MITH-94/11

\title{
A Possible Origin of the Wilson Lattice Fermion
}

\author{
Giuliano Preparata and She-Sheng Xue ${ }^{a)}$
}

INFN - Section of Milan, Via Celoria 16, Milan, Italy

\begin{abstract}
Considering relevant and irrelevant high-dimension operators for ordinary and mirror fermions respectively, we show that the Wilson lattice fermion can originate from a spontaneous symmetry breaking phenomenon. Ward identities, due to symmetries at the cutoff, guarantee the cancellation of mirror-fermion contributions and the infrared limit can be achieved. The Wilson parameter turns out to be fixed at $r \simeq 0.18$, where the vacuum energy of the system is minimized.
\end{abstract}

April, 1994

PACS 11.15Ha, 11.30.Rd, 11.30.Qc

a) E-mail address: xue@milano.infn.it 
1. The fermion "doubling" phenomenon is a well-known problem arising when fermion fields are defined on a lattice. In fact, this spectrum doubling is quite a general property of regularized fermion theories [1]]. However, this "problematic" phenomenon, instead of being a shortcoming of any reasonable regularization, perhaps gives us a physical hint to understand the short-distance behaviour of the Standard Model. Since chiral symmetry is perfectly conserved, the space-time lattice provides a very well-defined regularization for a fermionic field theory. In order for this occurrence, mirror fermions participate dynamically in the spectrum of the theory. In contrast, from a dynamical viewpoint, we have not completely understood the operator content of the theory at short distances, where there must exist local and non-local high-dimension operators for ordinary and mirror fermions and gauge bosons, owing to the experimental observation of a rich mass spectrum of fundamental particles in the low-energy region. At long distances, these high-dimension operators, however, should be relevant and irrelevant for ordinary fermions and mirror fermions respectively in such a way that mirror fermions decouple from the low-energy spectrum and ordinary fermions remain and couple properly with gauge bosons.

Let us consider lattice QCD as an example. It has been suggested that lattice regularization, avoiding fermion doubling, can be achieved by introducing an extra dimension-5 operator (the Wilson term) into the naive lattice fermion action $S_{d}(U, \bar{\psi}, \psi),\left(U \in S U\left(N_{c}\right)\right)$ [2],

$$
S=S_{d}(U, \bar{\psi}, \psi)+\frac{r}{a} \sum_{x, \mu} \bar{\psi}(x) \partial_{\mu}^{2} \psi(x),
$$

where $a$ is the lattice spacing and the lattice "laplacian" $\partial_{\mu}^{2}$ is defined as

$$
\partial_{\mu}^{2} \psi(x)=U_{\mu}(x) \psi\left(x+a_{\mu}\right)+U_{\mu}^{\dagger}(x) \psi\left(x-a_{\mu}\right)-2 \psi(x) .
$$

This extra term with a finite Wilson parameter $r(0<r \leq 1$ for reflection positivity) becomes an irrelevant operator for ordinary fermions in the infrared limit, while it is a relevant operator for mirror fermions in the high-energy regime, in fact it generates an effective mass $M \sim \frac{r}{a}$ for mirror fermions. If we wish to achieve a chiral-invariant QCD in the continuum limit, we must carefully tune only one free parameter in our lattice action so that intrinsic quark mass $m$ goes to zero as the continuum limit is approached. However, at the same time, the axial anomaly of the theory is restored [3] without any Goldstone modes, due to the explicit breaking of chiral symmetry in the Wilson term. In this letter, we would like to demonstrate that the Wilson fermion can be dynamically generated from chiral-invariant Nambu-Jona Lasinio 
(NJL) interactions [4] of ordinary and mirror fermions.

2. This attempt leads us to consider the following lagrangian,

$$
\begin{aligned}
S & =S_{g}(U)+S_{d}(\bar{\psi}, \psi, U)+S_{r}+S_{i r}, \\
S_{r} & =-G_{1} \sum_{x} \bar{\psi}_{L}(x) \cdot \psi_{R}(x) \bar{\psi}_{R}(x) \cdot \psi_{L}(x), \\
S_{i r} & =-\frac{G_{2}}{2} \sum_{\mu, x} \bar{\psi}_{L}(x) \cdot \partial_{\mu}^{2} \psi_{R}(x) \bar{\psi}_{R}(x) \cdot \partial_{\mu}^{2} \psi_{L}(x),
\end{aligned}
$$

where $S_{g}$ is the usual Wilson gauge-action and $G_{1,2}$ are two, as yet unspecified, Fermi-type $O\left(a^{2}\right)$ coupling constants. Note that (i) in the naive continuum limit $\left(S_{i r} \simeq 0\right.$ ), eq. (3) is just a gauged NJL model for ordinary fermions $(p a \ll 1)$ [5]; (ii) in the naive "lattice limit" $S_{i r}$ is a NJL interaction for mirror fermions $(p a \simeq 1)$ and $S_{r}$ is a NJL interaction in which both ordinary and mirror fermions participate; (iii) obviously, $S_{r}\left(S_{i r}\right)$ is a relevant (irrelevant) operator for ordinary fermions and both $S_{r}, S_{i r}$ are relevant operators for mirror fermions.

Using the Landau mean-field (tree-level) and the large- $N_{c}$ approach $\left(N_{c} \gg\right.$ $1, N_{c} G_{1,2}$ fixed), we consider the leading $O\left(N_{c}\right)$ terms of the gap equation for the fermion self-energy function $\Sigma(a p)$ :

$$
\Sigma(a p)=2 g_{1} \int_{l} \frac{\Sigma(l)}{\operatorname{Den}(l)}+\frac{g_{2}}{2} \int_{l} w(l) \frac{\Sigma(l)}{\operatorname{Den}(l)} w(a p)+\Sigma_{g}(a p),
$$

where $g_{1,2} a^{2}=N_{c} G_{1,2} ; l_{\mu}=q_{\mu} a, \int_{l}=\int_{-\pi}^{\pi} \frac{d^{4} l}{(2 \pi)^{4}} ; w(l)=\sum_{\mu}\left(1-\cos l_{\mu}\right)$ and $\operatorname{Den}(l)=$ $\sin ^{2} l_{\mu}+(a \Sigma(l))^{2} . \Sigma_{g}(a p)$ is the self-energy function due to the gauge interaction. On the basis of the structure of gap equation (6) and $\Sigma_{g}(a p)$ [3] one may rewrite $\Sigma(a p)=\Sigma^{\prime}(a p)+\bar{r} w(a p),(r=a \bar{r})$ and for arbitrary external momenta $p$, eq. (6) is equivalent to

$$
\begin{aligned}
\Sigma^{\prime}(a p) & =2 g_{1} \int_{l} \frac{\Sigma^{\prime}(l)+\bar{r} w(l)}{\operatorname{Den}(l)}+\Sigma_{g}(a p) ; \\
\bar{r} & =\frac{g_{2}}{2} \int_{l} w(l) \frac{\Sigma^{\prime}(l)+\bar{r} w(l)}{\operatorname{Den}(l)},
\end{aligned}
$$

where $\bar{r}$ is the following condensate of mirror fermions in the mean-field and large- $N_{c}$ approximation,

$$
\bar{r}=\frac{G_{2}}{4} \sum_{\mu, x}\left(\frac{1}{4}\right)\left\langle\bar{\psi}_{L}(x) \partial_{\mu}^{2} \psi_{R}(x)+\text { h.c. }\right\rangle .
$$

We clearly find that $\bar{r}$ stems from the contribution of mirror fermions owing to the factor $w(l)$ in eq. (8) and $\Sigma^{\prime}(a p)$ in eq. (7) received contributions from both ordinary and mirror fermions.

For small external momenta $p a \ll 1$ in eq. (6), we introduce an arbitrary scale $\epsilon(p a \ll \epsilon \ll \pi)$ separating the "lattice region" $(\epsilon, \pi)^{4}$, populated by mirror fermions, 
from the "continuum region" $(0, \epsilon)^{4}$, where ordinary fermions lie. For small internal momenta $l \in(0, \epsilon)^{4}, w(l) \simeq 0$ and $\bar{r} \rightarrow 0^{+}$(see eq. (8)), the gap equation (6) should reduce to its counterpart in the continuum theory. The mirror-fermion contributions to the gap-equation (6) come from the "lattice region", where internal momenta are large $l \in(\epsilon, \pi)$. Based on this separation, we write eq. (7) for $p a \ll 1$ as

$$
\Sigma(a p)=2 g_{1} \int_{l \in(0, \epsilon)} \frac{\Sigma^{\prime}(l)}{\operatorname{Den}(l)}+2 g_{1} \int_{l \in(\epsilon, \pi)} \frac{\Sigma^{\prime}(l)}{\operatorname{Den}(l)}+2 g_{1} \int_{l \in(\epsilon, \pi)} \frac{\bar{r} w(l)}{\operatorname{Den}(l)}+\Sigma_{g}(a p),
$$

where we have $\Sigma_{g}(a p)=\Sigma_{g}^{c}(a p)+\frac{\delta(r)}{a}$ [3], 7], where $\Sigma_{g}^{c}(a p)$ is the self-energy function $\Sigma_{g}(a p)$ in the "continuum region" and $\delta(r)$ is a finite function of $r$. The limit $\epsilon \rightarrow 0$ can be taken in the third term owing to the factor $w(l)$. The $\epsilon$-dependence of the first term must be cancelled by that of the second term in eq. (10). The value of $\epsilon$ is chosen so that in $\Sigma^{\prime}(l)$ we separate the $O\left(\frac{1}{a}\right)$ divergent part from the finite part $\Sigma_{c}(l)\left(a \Sigma_{c}(l) \ll 1\right)$, which is the counterpart of $\Sigma(l)$ in the continuum theory. One can thus rewrite eq. (10) as

$$
\Sigma_{c}(a p)=2 g_{1} \int_{l} \frac{\Sigma_{c}(l)}{\operatorname{Den}(l)}+\frac{\Delta}{a}+2 g_{1} \int_{l} \frac{\bar{r} w(l)}{\operatorname{Den}(l)}+\Sigma_{g}^{c}(a p)+\frac{\delta(r)}{a},
$$

where

$$
\frac{\Delta}{a} \simeq 2 g_{1} \int_{l \in(\epsilon, \pi)} \frac{\Sigma^{\prime}(l)}{\operatorname{Den}(l)}
$$

which is a mirror-fermion contribution via the NJL interaction $S_{r}$ in (3).

The symmetry of the action (3) implies Ward identities satisfied by Green functions on the lattice. These Ward identities must be obeyed up to powers of the lattice spacing $O(a)$. The Ward identity due to the chiral symmetry of the action (3) at the cutoff

$$
\left\langle\psi_{L}(0) \bar{\psi}_{R}(x)\right\rangle=0 \quad(x \gg a)
$$

implies that $O\left(\frac{1}{a}\right)$ divergences in eq. (11) should cancel

$$
\begin{aligned}
\Sigma_{c}(a p) & =2 g_{1} \int_{l} \frac{\Sigma_{c}(l)}{\operatorname{den}(l)}+\Sigma_{g}^{c}(a p) \\
0 & =\frac{\Delta}{a}+\frac{2 g_{1}}{a} \int_{l} \frac{r w(l)}{\operatorname{den}(l)}+\frac{\delta(r)}{a}
\end{aligned}
$$

where it is self-consistent that $\operatorname{den}(l)=\sin ^{2}(l)+\left(\Sigma_{c}(l) a+r w(l)\right)^{2}$. We find that $\frac{\Delta}{a}$ acts as the mass counterterm considered in the Rome approach $\mathbb{8}$, where the Ward identities are due to the BRST symmetry. Eq. (14) is analogous to the gap equation in the continuum theory [5] containing gauge and four-fermion interactions. In fact, the infrared scale $m=\Sigma_{c}(0)$, i.e., the v.e.v. of the Standard Model, and 
the mass counterterm $\frac{\Delta}{a}$ are contributions of ordinary fermions and mirror fermions respectively to the following condensate,

$$
M=-\frac{G_{1}}{2} \sum_{x}\langle\bar{\psi}(x) \psi(x)\rangle \simeq m+\frac{\Delta}{a} .
$$

It is clear that at short distances (the cutoff), the theory should recover the symmetries of action (3). This requires that all hard breaking terms $O\left(\frac{1}{a}\right)$, i.e., mirrorfermion contributions should cancel at the cutoff. Such cancellation is guaranteed by the Ward identities.

3. We now turn to the discussion of whether there exists a consistent solution of eqs. (8, 14, 15) in the infrared limit $\left(\Sigma_{c}(a p) a \ll 1\right)$. Since $\Sigma_{c}(a p) a \simeq 0$, eq. (8) approximately implies

$$
1=\frac{g_{2}}{2} \int_{l} w(l) \frac{w(l)}{\sin ^{2} l_{\mu}+(r)^{2} w(l)^{2}},
$$

establishing a relationship between $g_{2}$ and $r$, which is reported in Fig.(1). As one can see, we find that the coupling $g_{2}$ has to be strong enough $\left(g_{2}>g_{2}^{c} \simeq 0.2\right)$ in order for $r \sim O(1)$. Eq. (14) can be solved analytically. As discussed in refs. [6, 7], a critical line, where the infrared limit $\left(a \Sigma_{c}(a p) \ll 1\right)$ can be defined, is found in terms of $g_{1}$ and gauge coupling. The Ward identity (13) thus determines the mass counterterm $\frac{\Delta}{a}$ as a function of $g_{1}$ and gauge coupling.

Composite modes are bound to be produced once the spontaneous symmetry breakdown occurs $m \neq 0$, as evidenced by the non-trivial solutions of the gap equations (14). In order to see these modes, we calculate the four-fermion scattering amplitudes associated with the vertex $S_{r}$ within the approximation of $\Sigma_{c}(a p) \simeq m$ and neglecting gauge field. This calculation is straightforward and we just present the results. Composite modes in the pseudo-scalar channel $\Gamma_{p}\left(q^{2}\right)$ and the scalar channel $\Gamma_{s}\left(q^{2}\right)$ are

$$
\begin{aligned}
\Gamma_{p}\left(q^{2}\right) & =\frac{1}{2} \frac{1}{I_{p}(q) A\left(q^{2}\right)}, \\
\Gamma_{s}\left(q^{2}\right) & =\frac{1}{2} \frac{1}{\frac{4 N_{c}}{a^{2}} \int_{k} \frac{[m a+r w(k)]^{2}}{D(k, q a)}+I_{s}(q) A\left(q^{2}\right)},
\end{aligned}
$$

where

$$
\begin{aligned}
A\left(q^{2}\right) & =\sum_{\mu}\left(\frac{2}{a} \sin \frac{q_{\mu} a}{2}\right)^{2} \\
I_{p}(q) & =\frac{N_{c}}{4} \int_{k} \frac{c^{2}(k)+(r)^{2} s^{2}(k)}{D(k, q a)} \\
I_{s}(q) & =\frac{N_{c}}{4} \int_{k} \frac{c^{2}(k)}{D(k, q a)}
\end{aligned}
$$


where $c^{2}(k)=\sum_{\mu} \cos ^{2} k_{\mu}, s^{2}(k)=\sum_{\mu} \sin ^{2} k_{\mu}$ and $D(k, q a)=\operatorname{den}\left(k+\frac{q a}{2}\right) \operatorname{den}\left(k-\frac{q a}{2}\right)$. We find a massless Goldstone mode, which should be a candidate for the longitudinal mode of the massive gauge boson, and a scalar mode, which should be a candidate for the Higgs particle. The scalar mode disappears from the low-energy spectrum since its mass is proportional to $\frac{r}{a}$, a term which is contributed by mirror fermions.

4. In order to ascertain the stability, and hence the physical realizability, of the solution $\Sigma_{c}(a p) \neq 0$ and $r \neq 0$ to eqs. (8,14), we turn to the computation of the ground state energy. In the one-loop approximation $\left(O\left(N_{c}\right)\right), \Sigma_{c}(a p) \simeq m$ and neglecting the gauge field, the effective potential is given by

$$
V(M, \bar{r})=\frac{M^{2}}{G_{1}}+\frac{\bar{r}^{2}}{G_{2}}-N_{c} \operatorname{tr} \int_{l} \ln \left\{\frac{\gamma_{\mu} \sin l_{\mu}}{a}+(M+\bar{r} w(l))\right\}+\cdots,
$$

and the difference between the energy of the symmetric vacuum and broken vacuum $\Delta E_{\circ}=V(M, r)-V(0,0)$ is given by

$$
\Delta E_{\circ}=-\frac{2 N_{c}}{a^{4}} \int_{l} \sum_{k=1}^{\infty} \frac{1}{k+1}\left[\frac{(m a+r w(l))^{2}}{s^{2}(l)+(m a+r w(l))^{2}}\right]^{k+1},
$$

which is obtained from (21) by considering the gap equation (7),8) $(M \simeq \Sigma(l))$. This shows that the non-trivial solutions of the gap equations characterize a chirally asymmetric vacuum that has an energy density lower than that of the symmetric vacuum. However, $r$ gets the largest value permitted by eq. (8). On the other hand, noticing that composite bosons give a positive energy density of such broken "vacua" and this positive contribution certainly increases as $r^{2}$ increases, we turn to the computation of the total vacuum energy $(\Delta E)$ containing both fermion and composite boson contributions on the basis of the one-loop gap equations, (8. 14),

$$
\Delta E=-\left[\ln \int_{f} \exp \left(-S_{e f f}(m, r)\right)-\ln \int_{f} \exp \left(-S_{\text {eff }}(0,0)\right)\right],
$$

where $S_{\text {eff }}(m, r)$ is the effective Wilson action over the ground state. The details of the calculation are lengthy and will not be reported in this letter, we just present the result $\left(\Delta E_{\circ}\right.$ is $O\left(N_{c}\right)$ and the second term $O\left(N_{c}^{0}\right)$ ):

$$
\begin{aligned}
\Delta E \simeq & \Delta E_{\circ}-\left[1-e^{-\frac{\Delta E_{0}}{N_{c}}}\right] \\
& \cdot\left[-4+\int_{l}\left(4 g_{1} \tilde{\Gamma}_{s}(l)+\frac{1}{4 g_{1} \tilde{\Gamma}_{s}(l)}\right)+\int_{l}\left(4 g_{1} \tilde{\Gamma}_{p}(l)+\frac{1}{4 g_{1} \tilde{\Gamma}_{p}(l)}\right)\right],
\end{aligned}
$$

where

$$
\begin{aligned}
\tilde{\Gamma}_{p}(l) & =\frac{a^{2} I_{p}(l) A\left(l^{2}\right)}{4 N_{c}} \\
\tilde{\Gamma}_{s}(l) & =\frac{a^{2}}{4 N_{c}}\left(\frac{4 N_{c}}{a^{2}} \int_{k} \frac{[m a+r w(k)]^{2}}{D(k, l)}+I_{s}(l) A\left(l^{2}\right)\right) .
\end{aligned}
$$


Combining positive and negative contributions in eq. (24) and putting $m a \simeq 0$, $N_{c}=3$ and $g_{1} \simeq g_{1}^{c}(r)$ obtained from eq. (14) with $\Sigma_{g}^{c}(a p) \simeq 0$, we find (Fig.(2)) that $r \simeq 0.18$ is the energetically favoured solution. This shows that the effective Wilson action is stable over the chiral-symmetry-violating ground state.

5. The high-dimension operators $S_{r}$ and $S_{i r}$ at the cutoff (3) can probably be induced from the quantum gravity [9]. These operators evade in principle the "no-go" theorem. We have shown by considering the Dyson equation for the fermion sector and the vacuum energy that the operators $S_{r}$ and $S_{i r}$ can produce the low-energy spectrum $\Sigma_{c}(a p)$, the consistent mass counterterm $\frac{\Delta}{a}$ and the Wilson parameter $r$ that removes mirror fermions. Owing to the vector-like gauge symmetry of (3), the gauge field does not couple to the composite modes and the vacuum polarization is transverse and free from the $O\left(\frac{1}{a^{2}}\right)$ divergence. Logarithmic divergences in $\Sigma_{c}(a p)$, vacuum polarization and vertex function are treated in the normal renormalization prescription. As the ordinary-fermion mass $m$ is tuned to be zero, the axial anomaly is reproduced by $r \neq 0$ [3] without any extra massless Goldstone modes [10]. Thus in the context of a vectorial gauge theory with extended high-dimension operators (3), the "no-go" theorem is evaded also in practice.

As for a chiral gauge theory, it should be mentioned that the E-P model [11], which carefully breaks unwanted global symmetries, preserves the chiral gauge symmetries and gets rid of mirror fermions through the perturbation of four-fermi interactions. It was claimed [12 that the E-P model fails to reach its goal on the basis that the E-P model and the Smit-Swift model [13] are in the same universality class and spontaneous breaking of symmetry occurs. This "universality" [15] between the " $\bar{t} t$-condensate model" 16 and the Higgs- Yukawa model was shown based on renormalization group arguments (which however have been recently questioned [14 in the strong coupling region). According to the spirit of the approach presented in this paper, the key points for a possible lattice chiral gauge theory are: (i) whether there exist high-dimension operators $\left(S_{r}, S_{i r}\right.$ etc. $)$ that preserve chiral gauge symmetries at the cutoff, generating all necessary counterterms as carefully discussed in the Rome approach [8]; (ii) whether Ward identities stemming from symmetries at the cutoff (instead of the BRST symmetry implemented in the Rome approach) could consistently guarantee the achievement of a sensible low-energy chiral gauge theory. We are left with this problem for future work.

\section{References}

[1] H.B. Nielsen and M. Ninomiya, Nucl. Phys. B185 (1981) 20, ibid. B193 (1981) 173, Phys. Lett. B105 (1981) 219. 
[2] K. Wilson, in New phenomena in subnuclear physics (Erice, 1975) ed. A. Zichichi (Plenum, New York, 1977).

[3] L. H. Karsten and J. Smit, Nucl. Phys. B144 (1978) 536.

[4] Y. Nambu and G. Jona-Lasinio, Phys. Rev. 122 (1961) 345.

[5] W.A. Bardeen, C.T. Hill and S. Love, Phys. Rev. Lett. 56 (1986) 1230, Nucl. Phys. B273 (1986) 649.

[6] W.A. Bardeen, C.T. Hill and S. Love, Nucl. Phys. B323 (1989) 493,

W.A.Bardeen, S. T. Love and V.A. Miransky, Phys. Rev. D42 (1990) 3514.

[7] G. Preparata and S.-S. Xue, Phys. Lett. B302 (1993) 442;

S.-S. Xue, "Aspects of dynamical symmetry breaking on a lattice", heplat/9312027 and hep-lat/9402015.

[8] A. Borrelli, L. Maiani, G.C. Rossi, R. Sisto and M. Testa, Nucl. Phys. B333 (1990) 335; Phys. Lett. B221 (1989) 360.

[9] G. Preparata and S.-S. Xue, Phys. Lett. B264 (1991) 35; Nucl. Phys. B26 (Proc. Suppl.) (1992) 501; Nucl. Phys. B30 (Proc. Suppl.) (1993) 647.

[10] G. Preparata and S.-S. Xue, "The spontaneous breaking of chiral symmetry without Goldstone bosons", hep-lat/9401036.

[11] E. Eichten and J. Preskill, Nucl. Phys. B268 (1986) 179.

[12] M.F.L. Golterman, D.N. Petcher and E. Rivas, Nucl. Phys. B395 (1993) 597.

[13] J. Smit, Acta Physica Polonica B17 (1986) 531;

P.D.V. Swift, Phys. Lett. B145 (1984) 256.

[14] M. Lindner, Int. Mod. Phys. A8 (1993) 2167;

M. Gockeler, R. Horsley, P.E.L. Rakow, G. Schierholz and R. Sommer, Nucl. Phys. B371 (1992) 713;

A. Ali Khan, M. Gockeler, R. Horsley, P.E.L. Rakow, G. Schierholz and H. Stuben, FUB-HEP/93-14 and FUB-HEP/93-14.

[15] A. Hasenfratz, P. Hasenfratz, K. Jansen, J. Kuti and Yue Shen, Nucl. Phys. B365 (1991) 79.

[16] W.A. Bardeen, C. T. Hill and M. Lindner Phys. Rev. D41 (1990) 1647. 


\section{Figure Captions}

Figure 1: The function $r\left(g_{2}\right)$ in terms of $g_{2}\left(g_{2}>g_{2}^{c} \simeq 0.2\right)$.

Figure 2: The vacuum energy $\Delta E(r)$ in terms of the Wilson parameter $r$ $\left(r_{m} \simeq 0.18\right)$. 
This figure "fig1-1.png" is available in "png" format from: http://arxiv.org/ps/hep-lat/9312074v2 
This figure "fig1-2.png" is available in "png" format from: http://arxiv.org/ps/hep-lat/9312074v2 
This figure "fig1-3.png" is available in "png" format from: http://arxiv.org/ps/hep-lat/9312074v2 